PROCEEDINGS OF THE

AMERICAN MATHEMATICAL SOCIETY

Volume 137, Number 2, February 2009, Pages 699-709

S 0002-9939(08)09534-8

Article electronically published on September 3, 2008

\title{
ASYMPTOTIC STABILITY OF THE CROSS CURVATURE FLOW AT A HYPERBOLIC METRIC
}

\author{
DAN KNOPF AND ANDREA YOUNG
}

(Communicated by Richard A. Wentworth)

\begin{abstract}
We show that for any hyperbolic metric on a closed 3-manifold, there exists a neighborhood such that every solution of a normalized cross curvature flow with initial data in this neighborhood exists for all time and converges to a constant-curvature metric. We demonstrate that the same technique proves an analogous result for Ricci flow. Additionally, we prove shorttime existence and uniqueness of cross curvature flow under slightly weaker regularity hypotheses than were previously known.
\end{abstract}

It has been conjectured that any closed 3-manifold admitting a metric with negative sectional curvatures also admits a hyperbolic metric. This conjecture follows from Thurston's geometrization conjecture. As is well known, Ricci flow has shown itself to be a remarkably powerful tool in analyzing geometrization. However, it is not expected that Ricci flow can provide a straightforward proof of the hyperbolization conjecture. Indeed, Ricci flow will in general preserve negative sectional curvature only in dimension $n=2$. Moreover, one expects to see hyperbolic regions forming in a 3-manifold only at large times and typically after many topologically inessential surgeries. Consequently, there are motivations to explore alternative flows that may more readily yield information about negatively curved 3-manifolds, and in particular lead to a more direct proof of hyperbolization.

In 2004, Richard Hamilton and Bennett Chow proposed the cross curvature flow (XCF) for 3-manifolds and conjectured that it would preserve negative sectional curvature [3. They further conjectured that given any metric $g_{0}$ of strictly negative sectional curvatures on a closed manifold $\mathcal{M}^{3}$, one would obtain a 1-parameter family $g(t)$ of metrics evolved by normalized XCF such that the metrics $g(t)$ all have negative sectional curvatures and converge to a hyperbolic (constant curvature) metric as $t \rightarrow \infty$. An affirmative resolution of the Chow-Hamilton conjecture would imply that the space of hyperbolic metrics on a fixed manifold $\mathcal{M}^{3}$ is a deformation retract of the space of negatively curved metrics. This conjectural picture is in sharp contrast with the case in higher dimensions. Indeed, for $n \geq 4$, Gromov and Thurston have shown that there exist closed $n$-manifolds with sectional curvatures $-1-\varepsilon<K \leq-1$ that admit no metric of constant curvature $K=-1$ 8. More recently F. Thomas Farrell and Pedro Ontaneda have shown that in all dimensions $n \geq 10$, the space of negatively curved metrics on a closed manifold

Received by the editors July 16, 2007, and, in revised form, February 5, 2008 .

2000 Mathematics Subject Classification. Primary 53C44, 53C21, 58J35.

Key words and phrases. Cross curvature flow, asymptotic stability, hyperbolic metrics.

The first author was partially supported by NSF grants DMS-0505920 and DMS-0545984. 
$\mathcal{M}^{n}$ has infinitely many path components whenever it is nonempty [6]. Farrell and Ontaneda also provide examples of manifolds in dimensions $n \geq 10$ for which Ricci flow cannot deform all sufficiently pinched negatively curved metrics into constantcurvature metrics [5, 6].

In their seminal paper [3], Chow and Hamilton provide evidence in support of their conjecture. Let $E=\mathrm{Rc}-\frac{1}{2} R g$ denote the Einstein tensor and define a dual $(0,2)$-tensor $P$ by $P^{i j}=g^{i k} g^{j \ell} E_{k \ell}$. The functional

$$
J(g)=\int_{\mathcal{M}^{3}}\left\{\frac{1}{3} \operatorname{tr} P-(\operatorname{det} P)^{1 / 3}\right\} d \mu
$$

is nonnegative by the arithmetic-geometric mean inequality and vanishes if and only if $g$ is hyperbolic. Chow and Hamilton prove that $J(g(t))$ is nonincreasing for as long as a solution $g(t)$ of XCF exists. Their estimate is noteworthy because the effective volume $\int_{\mathcal{M}^{3}} \sqrt{\operatorname{det} P} d \mu$ is nondecreasing under XCF.

As of this writing, there are only a few other XCF results in the literature. John Buckland has established short-time existence of XCF for smooth initial data on compact manifolds; his approach uses De Turck diffeomorphisms to create a strictly parabolic system [2]. (XCF, like Ricci flow, is only weakly parabolic.) Several examples of solutions to XCF have been obtained by Dezhong Chen and Li Ma; these are warped product metrics on 2-torus and 2-sphere bundles over the circle 12 . Solutions on locally homogeneous manifolds 1 have recently been studied by Xiaodong Cao, Yilong Ni, and Laurent Saloff-Coste [4] and by David Glickenstein [7]. Also, in unpublished earlier work, Ben Andrews has obtained interesting estimates for more general solutions of XCF.

In contrast to Ricci flow, which is quasilinear, XCF is a fully nonlinear system. In spite of this increased complexity, we strongly believe that XCF is a highly promising tool for studying the geometric-topological properties of negatively curved 3-manifolds, and in particular the hyperbolization conjecture. In this short paper, we provide further evidence in support of this belief by establishing asymptotic stability of XCF. Namely, we show that for all initial data in a sufficiently small little-Hölder $C^{2+\rho}$ neighborhood of a metric of constant negative sectional curvature, the corresponding solution to a suitably normalized XCF will exist for all time and converge to a hyperbolic metric. (See Theorem 5.3 below.) To the best of our knowledge, these represent the first general long-time existence and stability theorems for XCF. We also prove short-time existence and uniqueness under weaker regularity hypotheses for initial data in an appropriate neighborhood of a hyperbolic metric. (See Theorem 4.1 below.) In ongoing work, we are pursuing further applications of these results.

This paper is organized as follows. In $\S 1$, we recall the definition of XCF and review some general theory regarding existence and stability of fully nonlinear equations. In $\S 2$, we review little-Hölder spaces. Our main computation is located in $\S 3$, where we linearize XCF, with a certain normalization, about a constant curvature metric. We answer the question of local existence and uniqueness in $\S 4$ and that of asymptotic stability in $\S 5$. Finally, in the appendix, we apply the same methods developed in this paper to verify asymptotic stability of Ricci flow at a hyperbolic

\footnotetext{
${ }^{1}$ In general, XCF can be defined in such a way that it is weakly parabolic if and only if the sectional curvatures of $\left(\mathcal{M}^{3}, g\right)$ all have the same sign. But on a homogeneous space, the flow reduces to an ODE system, so parabolicity is not an issue.
} 
metric. (The stability of Ricci flow with negatively curved initial data satisfying certain pinching hypotheses and other geometric bounds was studied by Rugang Ye in 1993 using alternate methods [13. His result is a priori stronger because it does not assume existence of a hyperbolic metric. On the other hand, the approach of this paper requires no diameter or volume hypotheses.)

\section{FUlly NONLINEAR EQUATIONS}

The cross curvature flow (XCF) is a fully nonlinear, weakly parabolic system of equations, which is defined as follows. As above, let $P$ denote the metric dual of the Einstein tensor. The cross curvature tensor is defined in local coordinates by

$$
X_{i j}=\frac{1}{2} P^{k \ell} R_{i k \ell j} .
$$

Notice that if we choose an orthonormal basis so that the eigenvalues of $P$ are $a=-R_{2332}, b=-R_{1331}$, and $c=-R_{1221}$, then the eigenvalues of $X$ are $-b c,-a c$, and $-a b$. In this definition, our sign convention is such that $R_{i j j i}(i \neq j)$ are the sectional curvatures, that is, $R_{i j k \ell}=g_{\ell m} R_{i j k}^{m}$. So if $\left(\mathcal{M}^{3}, g\right)$ has negative sectional curvatures, then $X$ is negative definite. One defines XCF for a manifold $\left(\mathcal{M}^{3}, g\right)$ of negative sectional curvatures by

$$
\begin{aligned}
\frac{\partial g}{\partial t} & =-2 X, \\
g(x, 0) & =g_{0}(x) .
\end{aligned}
$$

(The opposite sign convention is used on a manifold of positive sectional curvatures.)

Now let us establish some notation useful for studying fully nonlinear systems in general. Let $I$ be an interval and let $\mathbb{X}$ be a Banach space with norm $\|\cdot\|=\|\cdot\|_{\mathbb{X}}$. For a linear operator $A: D(A) \subset \mathbb{X} \rightarrow \mathbb{X}$, we define the graph norm to be

$$
\|x\|_{A}=\|x\|+\|A x\|
$$

and we let

$$
\|A\|_{L(D(A), \mathbb{X})}=\sup _{\|x\|_{D(A)}=1}\|A x\|_{\mathbb{X}} .
$$

We denote the spaces of continuous and $m$ times continuously differentiable functions $f: I \rightarrow \mathbb{X}$ as $C(I ; \mathbb{X})$ and $C^{m}(I ; \mathbb{X})$ with the usual norms. We also have weighted spaces $B_{\mu}((a, b] ; \mathbb{X})$ and $C_{\alpha}^{\alpha}((a, b] ; \mathbb{X})$ of functions that are bounded and Hölder continuous on $[a+\epsilon, b]$ but not necessarily up to $t=a$.

Specifically, let $\mu \in \mathbb{R}$ and define

$$
B_{\mu}((a, b] ; \mathbb{X}):=\left\{f:(a, b] \rightarrow \mathbb{X}:\|f\|_{B_{\mu}((a, b] ; \mathbb{X})}:=\sup _{a<t \leq b}(t-a)^{\mu}\|f(t)\|<\infty\right\} .
$$

Let $[f]_{C^{\alpha}([a, b] ; \mathbb{X})}=\sup _{a<s<t<b} \frac{\|f(t)-f(s)\|}{(t-s)^{\alpha}}$ denote the usual $C^{\alpha}$ seminorm. Then, for $0<\alpha<1, C_{\alpha}^{\alpha}((a, b] ; \mathbb{X})$ is the set of bounded functions $f:(a, b] \rightarrow \mathbb{X}$ such that

$$
[f]_{C_{\alpha}^{\alpha}((a, b] ; \mathbb{X})}:=\sup _{0<\epsilon<b-a} \epsilon^{\alpha}[f]_{C^{\alpha}([a+\epsilon, b] ; \mathbb{X})}<\infty,
$$

with norm $\|f\|_{C_{\alpha}^{\alpha}((a, b] ; \mathbb{X})}:=\sup _{a<t \leq b}\|f(t)\|+[f]_{C_{\alpha}^{\alpha}((a, b] ; \mathbb{X})}$.

We employ the theory collected in [11] regarding the local existence, uniqueness, and asymptotic behavior of solutions of fully nonlinear parabolic equations. Let 
$\mathbb{D}$ be a Banach space continuously embedded in $\mathbb{X}$ and having norm $\|\cdot\|_{\mathbb{D}}$. We consider the initial value problem

$$
\begin{aligned}
& u^{\prime}(t)=F(u), \quad t>0, \\
& u(0)=u_{0},
\end{aligned}
$$

where $F: \mathcal{O} \rightarrow \mathbb{X}$ for $\mathcal{O}$ an open subset of $\mathbb{D}$. We make several assumptions about $F$ that we will verify in $\S 3$ below.

(1) $F$ is continuous and Fréchet differentiable with respect to $u$.

(2) The derivative $F_{u}$ is sectorial in $\mathbb{X}$; i.e. there are constants $\omega \in \mathbb{R}, \theta \in$ $\left(\frac{\pi}{2}, \pi\right), M>0$ such that $\rho\left(F_{u}\right) \supset S_{\theta, \omega}=\{\lambda \in \mathbb{C}: \lambda \neq \omega,|\arg (\lambda-\omega)|<\theta\}$ and

$$
\left\|R\left(\lambda, F_{u}\right)\right\|_{L(\mathbb{X}, \mathbb{X})} \leq \frac{M}{|\lambda-\omega|}
$$

for all $\lambda \in S_{\theta, \omega}$. Here $\rho\left(F_{u}\right)$ denotes the resolvent set of $F_{u}$ and $R\left(\lambda, F_{u}\right)=$ $\left(\lambda I-F_{u}\right)^{-1}$ is the resolvent operator.

(3) $F_{u}$ has its graph norm equivalent to the norm of $\mathbb{D}$.

(4) Let $\bar{u} \in \mathcal{O}$. Then there exist $r, C$ depending on $\bar{u}$ such that, for all $u, v, w \in$ $B(\bar{u}, r)$,

$$
\left\|F_{u}(v)-F_{u}(w)\right\|_{L(\mathbb{D}, \mathbb{X})} \leq C|| v-w \|_{\mathbb{D}},
$$

where $B(\bar{u}, r)$ denotes the ball around $\bar{u} \in \mathcal{O}$ of radius $r$ measured with respect to the $\mathbb{D}$ norm.

For such $F$, we have the following local existence and uniqueness theorem.

Theorem 1.1 ([11, Theorem 8.1.1]). Let $F(\bar{u}) \in \overline{\mathbb{D}}$. Then there exist $\delta, r>0$, depending on $\bar{u}$, such that for all $u_{0} \in B(\bar{u}, r) \subset \mathbb{D}$ with $F\left(u_{0}\right) \in \overline{\mathbb{D}}$, there exists a solution $u$ to (1.3) such that $u \in C([0, \delta] ; \mathbb{D}) \cap C^{1}([0, \delta] ; \mathbb{X})$. Furthermore, $u \in$ $C_{\alpha}^{\alpha}((0, \delta))$ and $\lim _{\epsilon \rightarrow 0} \epsilon^{\alpha}[u]_{C^{\alpha}([\epsilon, 2 \epsilon] ; \mathbb{D})}=0$. Finally, $u$ is the unique solution of (1.3) in $\bigcup_{0<\beta<1} C_{\beta}^{\beta}((0, \delta] ; \mathbb{D}) \cap C([0, \delta] ; \mathbb{D})$.

We would additionally like to consider the asymptotic behavior of (1.3). Notice that we can linearize this problem around a stationary solution $u$ and rewrite it as

$$
\begin{aligned}
& \bar{u}^{\prime}(t)=A \bar{u}(t)+G(\bar{u}(t)-u), \quad t>0, \\
& \bar{u}(0)=\bar{u}_{0},
\end{aligned}
$$

where $A=F_{u}(\bar{u})$ and $G(\bar{u}-u)=F(\bar{u})-A \bar{u}$. Notice that $F$ fully nonlinear means that $G$ contains "top order" terms. We can assume $F(u)=0$. We would like $A$ to be sectorial, to have graph norm equivalent to that of $\mathbb{D}$, and for the spectrum of $A$ to satisfy

$$
\sup \{\Re(\lambda): \lambda \in \sigma(A)\}=-\omega_{0}<0 .
$$

We also want $G$ to be Fréchet differentiable with a locally Lipschitz continuous derivative and such that

$$
G(\bar{u}-u)=0, \quad G^{\prime}(\bar{u}-u)=0 .
$$

Then we have the following stability result.

Theorem 1.2. Let $\omega \in\left[0, \omega_{0}\right)$, and let $F\left(\bar{u}_{0}\right) \in \overline{\mathbb{D}}$. There exist $r, C>0$ such that for all $\bar{u}_{0} \in B(u, r) \subset \mathbb{D}$ the solution $\bar{u}\left(t ; \bar{u}_{0}\right)$ of (1.3) exists for all time and

$$
\|\bar{u}(t)-u\|_{\mathbb{D}}+\left\|\bar{u}^{\prime}(t)\right\|_{\mathbb{X}} \leq C e^{-\omega t}\left\|\bar{u}_{0}\right\|_{\mathbb{D}},
$$

for $t \geq 0$. 


\section{LitTLE-HÖLDER SPACES}

Let $\mathcal{M}^{3}$ denote a compact manifold admitting a hyperbolic metric $g$. Fix a background metric $\hat{g}$ and a finite atlas $\left\{U_{v}\right\}_{1 \leq v \leq \Upsilon}$ of coordinate charts covering $\mathcal{M}^{3}$. For each $r \in \mathbb{N}$ and $\rho \in(0,1]$, let $\mathfrak{h}^{r+\rho}$ denote the little-Hölder space of symmetric $(2,0)$-tensors with norm $\|\cdot\|_{r+\rho}$ derived from

$$
\|u\|_{0+\rho}:=\max _{\substack{1 \leq i, j \leq 3 \\ 1 \leq v \leq \Upsilon}}\left(\sup _{x \in U_{v}}\left|u_{i j}(x)\right|+\sup _{x, y \in U_{v}} \frac{\left|u_{i j}(x)-u_{i j}(y)\right|}{\left(d_{\hat{g}}(x, y)\right)^{\rho}}\right) .
$$

It is well known that different choices of background metrics or atlases give equivalent norms.

Henceforth fix $\rho \in(0,1)$. For the remainder of the paper, we will let

$$
\mathbb{D}=\mathfrak{h}^{2+\rho} \quad \text { and } \quad \mathbb{X}=\mathfrak{h}^{0+\rho} .
$$

Then $\mathbb{D} \hookrightarrow \mathbb{X}$ is a continuous and dense inclusion. Notice that these spaces are the closure under $\|\cdot\|_{2+\rho}$ and $\|\cdot\|_{0+\rho}$ respectively of the space of $C^{\infty}$ sections of the bundle $S_{2}\left(\mathcal{M}^{3}\right)$ of symmetric $(2,0)$-tensors over $\mathcal{M}^{3}$. (Recall that smooth sections are not dense in the usual Hölder spaces.)

\section{A MOdified CRoss CURVATURE FLOW}

For what follows, we consider a certain normalization of cross curvature flow which we call KXCF. It is defined by

$$
\frac{\partial \bar{g}}{\partial t}=-2 X(\bar{g})-2 K^{2} \bar{g} .
$$

Notice that a hyperbolic metric $g$ of constant curvature $K<0$ is a fixed point of this flow. Such a metric is also a fixed point of the volume-normalized cross curvature flow (NXCF) defined to be

$$
\frac{\partial \bar{g}}{\partial t}=-2 X(\bar{g})+\frac{2}{3} x \bar{g}
$$

where $x=\frac{\int_{\mathcal{M}} \operatorname{tr}_{g} X d \mu}{\int_{\mathcal{M}} d \mu}$. Notice that (3.2) has a nonlocal term on the right-hand side. Since both KXCF and NXCF are equivalent to XCF via a reparameterization of space and time, we prefer to use the former.

Lemma 3.1. KXCF differs from XCF only by a change of scale in space and time.

Proof. Define dilating factors $\psi(t)>0$ by $\psi(t)=A e^{2 K^{2} t}$ and define $\tilde{t}=\int_{0}^{t} \psi^{2}(\tau) d \tau$ so that $\frac{d \tilde{t}}{d t}=\psi^{2}(t)$. If we let $\tilde{g}=\psi \bar{g}$, then $X(\tilde{g})=\frac{1}{\psi} X(\bar{g})$. Supposing $\bar{g}$ solves (3.1), we have the following computation:

$$
\begin{aligned}
\frac{\partial \tilde{g}}{\partial \tilde{t}}=\frac{d t}{d \tilde{t}}\left(\frac{\partial}{\partial t}(\psi \bar{g})\right) & =\frac{d t}{d \tilde{t}}\left(\frac{\partial \psi}{\partial t} \bar{g}\right)+\frac{d t}{d \tilde{t}}\left(\psi\left(-2 X(\bar{g})-2 K^{2} \bar{g}\right)\right) \\
& =\frac{1}{\psi^{3}} \frac{\partial \psi}{\partial t} \tilde{g}-2 X(\tilde{g})-\frac{2}{\psi^{2}} K^{2} \tilde{g} \\
& =-2 X(\tilde{g}) .
\end{aligned}
$$

Thus $\tilde{g}$ solves (1.2), and we have shown the desired equivalence. 
We now want to define a DeTurck-modified cross curvature flow

$$
\frac{\partial}{\partial t} \bar{g}(x, t)=F(x, \bar{g}(x, t))
$$

for Riemannian metrics $\bar{g}(\cdot, t)$ in a neighborhood $\mathcal{O} \subset \mathbb{D}$ of the hyperbolic metric $g$ on $\mathcal{M}^{3}$. Here $\mathcal{O}$ is an open set in $\mathbb{D}$ to be determined below.

Given $\bar{g} \in \mathcal{O}$ and a smooth section $h$ of $S_{2}\left(\mathcal{M}^{3}\right)$, define a vector field $Y(\bar{g}, h)$ on $\mathcal{M}^{3}$ in local coordinates by

$$
Y^{\ell}(\bar{g}, h):=\frac{1}{2} \bar{g}^{k \ell} \partial_{k}\left(\bar{g}^{i j} h_{i j}\right)-\bar{g}^{k \ell} \bar{g}^{i j} \bar{\nabla}_{i} h_{j k}
$$

Assume that $g$ has constant sectional curvature $K<0$, and consider the DeTurck cross curvature flow (DXCF) given by

$$
\begin{aligned}
\frac{\partial}{\partial t} \bar{g} & =F(\bar{g}):=-2 X(\bar{g})+K \mathcal{L}_{Y(g, \bar{g})} g-2 K^{2} \bar{g}, \\
\bar{g}(0) & =g_{0} .
\end{aligned}
$$

Notice that $F(g)=0$.

We next derive the linearization of DXCF.

Lemma 3.2. If $\tilde{g}=\bar{g}+h$, the Fréchet derivative $F_{\bar{g}}$ is the linear operator $A_{\bar{g}}$ given by

$$
\begin{aligned}
\left(A_{\bar{g}} h\right)_{i k} & =\frac{1}{2} \bar{R}_{j i k}^{\ell}\left\{\bar{\Delta}_{\ell} h_{\ell}^{j}+\left(\mathcal{L}_{Y(\bar{g}, h)} \bar{g}\right)_{\ell}^{j}\right\}-\frac{1}{4} \bar{R}\left\{\bar{\Delta}_{\ell} h_{i k}+\left(\mathcal{L}_{Y(\bar{g}, h)} \bar{g}\right)_{i k}\right\} \\
& +\frac{1}{2} \bar{R}_{\ell}^{j}\left(\bar{\nabla}_{i} \bar{\nabla}_{k} h_{j}^{\ell}-\bar{\nabla}_{j} \bar{\nabla}_{k} h_{i}^{\ell}-\bar{\nabla}_{i} \bar{\nabla}^{\ell} h_{j k}+\bar{\nabla}_{j} \bar{\nabla}^{\ell} h_{i k}\right) \\
& -\frac{1}{2} \bar{R}_{i k}\left(\bar{\Delta} H-\bar{\delta}^{2} h\right)+K\left(\mathcal{L}_{Y(\bar{g}, h)} \bar{g}\right)_{i k}-2 K^{2} h \\
& -\bar{R}_{i j k}^{\ell} \bar{R}_{\ell}^{m} h_{m}^{j}+\frac{1}{2} \bar{R}_{i j m}^{\ell} \bar{R}_{\ell}^{j} h_{k}^{m}-\frac{1}{2} \bar{R}_{i j k}^{m} \bar{R}_{\ell}^{j} h_{m}^{\ell}-\frac{1}{2}\langle\bar{R} \mathrm{c}, h\rangle_{\bar{g}} \bar{R}_{i k} .
\end{aligned}
$$

At a metric $g$ of constant sectional curvature $K<0$, one has

$$
R_{i j k \ell}=K\left(g_{i \ell} g_{j k}-g_{i k} g_{j \ell}\right), \mathrm{Rc}=2 K g, \text { and } R=6 K
$$

whence the formula above reduces to

$$
A_{g} h=-K \Delta h-2 K^{2} H g+2 K^{2} h
$$

where $H=g^{i j} h_{i j}$.

Proof. Using the definition of the cross curvature tensor, $X_{i j}=\frac{1}{2} P^{k \ell} R_{i k \ell j}$, one linearizes using standard first variation formulas, as found for instance in [1] or [9].

Observe that $A_{g}$ is a self-adjoint elliptic operator. The $L^{2}$ spectrum of $A_{g}$ consists of discrete eigenvalues of finite multiplicity contained in the half-line $\left(-\infty, 2 K^{2}\right]$ and accumulating only at $-\infty$. Standard Schauder theory implies that $A_{g}$ is sectorial with its graph norm equivalent to $\|\cdot\|_{2+\rho}$. In particular, there exists $C \in(0, \infty)$ such that

$$
\frac{1}{C}\|h\|_{A_{g}} \leq\|h\|_{2+\rho} \leq C\|h\|_{A_{g}} .
$$
form

Noting that $\Delta_{\ell} h=\Delta h+H \mathrm{Rc}-R h$ on $\left(\mathcal{M}^{3}, g\right)$, one may also write $A_{g}$ in the

$$
A_{g} h=-K\left(\Delta_{\ell} h+4 K h\right) .
$$




\section{Local EXistence AND UniQueness}

Recall our notation that $\mathbb{D}=\mathfrak{h}^{2+\rho}$ and $\mathbb{X}=\mathfrak{h}^{0+\rho}$. Let $\bar{g} \in \mathbb{D}$. In each coordinate chart $U_{v}$, one may write

$$
\left(A_{\bar{g}} h\right)_{i j}=a^{k \ell} \partial_{k} \partial_{\ell} h_{i j}+b^{k} \partial_{k} h_{i j}+c_{i j}^{k \ell} h_{k \ell},
$$

where $a, b$, and $c$ depend on $x \in U_{v}$ and $\bar{g}, \partial \bar{g}, \partial^{2} \bar{g}$. By taking $\bar{g}$ close enough to $g$ in $\mathbb{D}$, we can make $a, b, c$ as close in $L^{\infty}$ as desired to their values for $A_{g}$.

Let $\mathcal{O}:=B_{\eta}^{2+\rho}(g)$, where $B_{\eta}^{2+\rho}(g)$ denotes the $\eta$-ball around $g$; i.e.

$$
B_{\eta}^{2+\rho}(g):=\left\{\bar{g} \in \mathfrak{h}^{2+\rho}:\|\bar{g}-g\|_{2+\rho}<\eta\right\} .
$$

Choose $\eta>0$ small enough such that for all $\bar{g} \in \mathcal{O}$,

(1) $\bar{g}$ is a Riemannian metric,

(2) $A_{\bar{g}}$ is uniformly elliptic, and

(3) there exists a sufficiently small $\delta>0$, to be chosen below, such that $\left\|\left(A_{\bar{g}}-A_{g}\right) h\right\|_{0+\rho}<\delta\|h\|_{2+\rho}$.

Let $\delta=(M+1)^{-1}$, with $M$ as in (1.4) depending only on the maximum of the resolvent operator. Then it is a standard fact that $A_{\bar{g}}$ is sectorial for all $\bar{g} \in \mathcal{O}$. (For example, see [11, Proposition 2.4.2].) We can then choose $\delta$ smaller if necessary (depending on $C$ in (3.5) ) so that the graph norm of $A_{\bar{g}}$ is equivalent to $\|\cdot\|_{2+\rho}$.

Let

$$
G(h)=F(g+h)-A_{g} h=-2 X(g+h)+K \mathcal{L}_{Y(g, g+h)} g-2 K^{2}(g+h)-A_{g} h .
$$

Since $g$ is a fixed point of (3.1), and $A_{g}$ is linear, we see that $G(0)=0$. From the computation above, it is clear that the derivative of $G$ at $h$ is

$$
G_{h} k=A_{g+h} k-A_{g} k,
$$

so $G^{\prime}(0)=0$ as well. The fact that for any $r \in(0, \eta]$ there exists $C>0$ such that $\left\|G_{h} z\right\|_{\mathbb{X}} \leq C\|z\|_{\mathbb{D}}$ uniformly for $h \in B_{r}^{2+\rho}(0)$ follows from property (3) above. This establishes the local Lipschitz continuity that we need to apply Theorem 1.2 .

Given $\bar{g} \in \mathcal{O}$, choose $\varepsilon>0$ small enough that $B_{\varepsilon}^{2+\rho}(\bar{g}) \subseteq \mathcal{O}$. Fix any coordinate chart $U_{v}$. Given $u \in B_{\varepsilon}^{2+\rho}(\bar{g})$, let $a(x) \equiv a\left(x, u, \partial u, \partial^{2} u\right), b(x) \equiv b\left(x, u, \partial u, \partial^{2} u\right)$, and $c(x) \equiv c\left(x, u, \partial u, \partial^{2} u\right)$ denote the local coefficients of $A_{u}$, as in (4.1). Then for any $h \in \mathbb{D}$, one has

$$
\begin{aligned}
\frac{\left|a^{k \ell}(x) \partial_{k} \partial_{\ell} h_{i j}(x)-a^{k \ell}(y) \partial_{k} \partial_{\ell} h_{i j}(y)\right|}{\left(d_{\hat{g}}(x, y)\right)^{\rho}} & \leq\left|a^{k \ell}(x) \frac{\partial_{k} \partial_{\ell} h_{i j}(x)-\partial_{k} \partial_{\ell} h_{i j}(y)}{\left(d_{\hat{g}}(x, y)\right)^{\rho}}\right| \\
& +\left|\partial_{k} \partial_{\ell} h_{i j}(y) \frac{a^{k \ell}(x)-a^{k \ell}(y)}{\left(d_{\hat{g}}(x, y)\right)^{\rho}}\right| \\
& \leq\|u\|_{2+0}\|h\|_{2+\rho}+\|h\|_{2+0}\|u\|_{2+\rho}
\end{aligned}
$$

Similar arguments apply to the lower order terms. For the details, one need only make minor modifications to the proof of Lemma 3.3 in $[9$. In this way, it is easy to see that

$$
\left\|A_{u}(v)-A_{u}(w)\right\|_{0+\rho} \leq C\|u\|_{2+\rho}\|v-w\|_{2+\rho}
$$

for all $u, v, w \in B_{\varepsilon}^{2+\rho}(\bar{g})$.

Then we can apply Theorem 1.1 to obtain the following theorem. 
Theorem 4.1. Let $\left(\mathcal{M}^{3}, g\right)$ be a Riemannian manifold having constant sectional curvature $K<0$. There exist $\delta, r>0$ such that for all $\bar{g}_{0} \in B_{r}^{2+\rho}(g)$ there exists a solution $\bar{g} \in C\left([0, \delta] ; \mathfrak{h}^{2+\rho}\right) \cap C^{1}\left([0, \delta] ; \mathfrak{h}^{0+\rho}\right)$ of DXCF that exists for all $t \in[0, \delta]$. This is the unique solution in $\bigcup_{0<\beta<1} C_{\beta}^{\beta}\left(\left(t_{0}, t_{0}+\delta\right] ; \mathfrak{h}^{2+\rho}\right) \cap C\left(\left[t_{0}, t_{0}+\delta\right] ; \mathfrak{h}^{2+\rho}\right)$.

Remark 4.2. The idea of using a DeTurck trick to prove short-time existence and uniqueness for XCF is due to Buckland [2]. Because of the invariance of the Riemann curvature tensor under the infinite-dimensional diffeomorphism group, the symbol of the linearization of (1.2) is only degenerate elliptic. Indeed, Buckland shows that, in appropriate coordinates, the symbol of the linearization, acting on a vector $\left(h_{11}, h_{12}, h_{13}, h_{22}, h_{33}, h_{23}\right)^{T}$ representing the components of a variation, is represented by the matrix

$$
\left(\begin{array}{cccccc}
0 & 0 & 0 & \Lambda^{22} & \Lambda^{33} & 2 \Lambda^{23} \\
0 & 0 & 0 & -\Lambda^{12} & 0 & -\Lambda^{13} \\
0 & 0 & 0 & 0 & -\Lambda^{13} & -\Lambda^{12} \\
0 & 0 & 0 & \Lambda^{11} & 0 & 0 \\
0 & 0 & 0 & 0 & \Lambda^{11} & 0 \\
0 & 0 & 0 & 0 & 0 & \Lambda^{11}
\end{array}\right)
$$

with eigenvalues $\Lambda^{11}>0$ and 0 , each of multiplicity three. See [2, equation (4)]. Ellipticity fails because of the null eigenvalue. The DeTurck diffeomorphisms effectively fix a gauge, breaking diffeomorphism invariance and making the linearization strongly elliptic. In this way, Buckland proves short-time existence and uniqueness for smooth initial data. The theorem above may be regarded as a mild extension of his result, in the sense that it proves existence and uniqueness of solutions to DXCF for somewhat less regular initial data, at least for such data sufficiently near a hyperbolic metric.

\section{Stability}

Without loss of generality, we may assume that $\left(\mathcal{M}^{3}, g\right)$ has constant sectional curvature $K=-1$. Henceforth write $A \equiv A_{g}$, noting that

$$
\begin{aligned}
A h & =\Delta_{\ell} h-4 h \\
& =\Delta h-2 H g+2 h .
\end{aligned}
$$

Clearly, the $L^{2}$ spectrum of $A$ is contained in $\left(-\infty, \omega_{0}\right]$ for some $\omega_{0} \leq 2$. We now further analyze the spectrum, using a Bochner formula due to Koiso 10. Notice that, for $h$ a symmetric $(2,0)$-tensor on a closed manifold $\left(\mathcal{M}^{n}, g\right)$, one has

$$
\|\nabla h\|^{2}=\|\delta h\|^{2}+\frac{1}{2}\|T\|^{2}+\int_{\mathcal{M}^{n}}\left(R_{i j k l} h^{i l} h^{j k}-R_{i}^{k} h_{j k} h^{i j}\right) d \mu,
$$

where $T=T(h)$ is a $(3,0)$-tensor defined by $T_{i j k}=\nabla_{k} h_{i j}-\nabla_{i} h_{j k}$ and $(\delta h)_{k}=$ $-g^{i j} \nabla_{i} h_{j k}$. In our case, $g$ has constant sectional curvature $K<0$, so again we use the formulas $R_{i j k \ell}=K\left(g_{i \ell} g_{j k}-g_{i k} g_{j \ell}\right)$ and Rc $=2 K g$. Thus Koiso's Bochner formula reduces to

$$
\|\nabla h\|^{2}=\|\delta h\|^{2}+\frac{1}{2}\|T\|^{2}-\|H\|^{2}+3\|h\|^{2} .
$$

This observation implies that

$$
\int(A h, h) d \mu \leq-\|H\|^{2}-\|h\|^{2} \leq-\|h\|^{2}<0 .
$$


Thus there exists an $\omega \geq 1$ such that the $L^{2}$ spectrum of $A_{g}$ is contained in the half-line $(-\infty,-\omega]$. So we can apply Theorem 1.2 to obtain asymptotic stability for DXCF.

Finally, we shall show that asymptotic stability for DXCF implies the same for XCF. We will utilize the following lemma, whose proof may be found in $[9]$.

Lemma 5.1. Let $Y(t)$ be a vector field on a Riemannian manifold $\left(\mathcal{M}^{n}, g(t)\right)$, where $0 \leq t<\infty$, and suppose there are constants $0<c \leq C<\infty$ such that

$$
\sup _{x \in M^{n}}|Y(x, t)|_{g(t)} \leq C e^{-c t} .
$$

Then the diffeomorphisms $\varphi_{t}$ generated by $Y$ converge exponentially to a fixed diffeomorphism $\varphi_{\infty}$ of $\mathcal{M}^{n}$.

Lemma 5.2. Let $g$ be a metric of constant negative sectional curvature on $\mathcal{M}^{3}$. Suppose there exists an $r$ such that for all $\tilde{g}_{0} \in B_{r}^{2+\rho}(g)$, the unique solution $\bar{g}(t)$ of (3.4) with $\bar{g}(0)=\tilde{g}_{0}$ converges exponentially fast to $g$. Then the unique solution $\tilde{g}(t):=\varphi_{t}^{*} \bar{g}$ of (3.1) with $\tilde{g}(0)=\tilde{g}_{0}$ converges exponentially fast to a constant curvature metric $\tilde{g}_{\infty}$.

Proof. Recall that $Y$ is defined to be

$$
Y^{l}=\frac{1}{2} \bar{g}^{k l} \partial_{k}\left(g^{i j} \bar{g}_{i j}\right)-g^{k l} g^{i j} \nabla_{i} \bar{g}_{j k} .
$$

Since $\bar{g}(t) \rightarrow g$ exponentially fast, we have $Y^{l} \rightarrow 0$ exponentially fast as well. So Lemma[5.1 implies that the diffeomorphisms $\varphi_{t}$ converge to a fixed diffeomorphism $\varphi_{\infty}$. Thus $\tilde{g}(t)$ converges to a limit metric $\tilde{g}_{\infty}$, which by diffeomorphism invariance has constant sectional curvature.

Then we can apply Theorem 1.2 to obtain asymptotic stability of KXCF.

Theorem 5.3. Let $\left(\mathcal{M}^{3}, g\right)$ be a closed Riemannian manifold with constant sectional curvature $K<0$. Then there exists $\delta>0$ such that for all $\bar{g}_{0} \in B_{\delta}^{2+\rho}(g)$, the solution $\bar{g}$ to (3.1) having initial condition $\bar{g}_{0}$ exists for all time and converges exponentially fast to a constant curvature hyperbolic metric.

\section{Appendix A. Asymptotic stability of Ricci flow AT A HYPERBOLIC METRIC}

The methods developed in this paper provide a simple proof of the asymptotic stability of Ricci flow at a hyperbolic metric. As noted above, a more powerful stability result was obtained earlier by Ye using somewhat different methods [13. Recall that the Ricci flow is defined to be

$$
\begin{aligned}
\frac{\partial \bar{g}}{\partial t} & =-2 \operatorname{Rc}(\bar{g}), \\
\bar{g}(0) & =\bar{g}_{0} .
\end{aligned}
$$

We proceed as above and define a normalized Ricci flow (KNRF) that differs from the usual volume-normalized flow but which also can be obtained from Ricci flow only by a reparameterization of space and time. KNRF is given by

$$
\begin{aligned}
\frac{\partial \bar{g}}{\partial t} & =-2 \operatorname{Rc}(\bar{g})+4 K \bar{g}, \\
\bar{g}(0) & =\bar{g}_{0} .
\end{aligned}
$$


In particular, a constant curvature metric with $K<0$ is a fixed point of KNRF.

Using standard variation formulas, we can linearize the right-hand side of the KNRF equation about a hyperbolic metric $g$ having constant curvature $K=-1$ to obtain

$$
A_{g} h=\Delta_{\ell} h+4 K h=\Delta h-2 H g+2 h .
$$

The same trick as above allows us to bound the spectrum in the interval $(\infty,-1]$. By mimicking our previous analysis, one easily checks that the hypotheses of Theorem 1.2 are satisfied. (Or see the detailed calculations in [9], which treat a more technically difficult case in which there is a center manifold present.) Thus we obtain the following theorem.

Theorem A.1. Let $\left(\mathcal{M}^{3}, g\right)$ be a closed Riemannian manifold having constant sectional curvature $K<0$. Then there exists $\delta>0$ such that for all $\bar{g}_{0} \in B_{\delta}^{2+\rho}(g)$, the solution $\bar{g}$ to (A.2) having initial condition $\bar{g}_{0}$ exists for all time and converges exponentially fast to a constant curvature hyperbolic metric.

Notice that curvature pinching is not quite sufficient to apply this theorem: one also requires a Hölder bound on the first derivative of the curvature. Compare [5].

\section{ACKNOWLEDGMENT}

The authors thank the referee for making helpful suggestions to improve the exposition of this paper.

\section{REFERENCES}

1. Besse, Arthur L. Einstein Manifolds. Ergebnisse der Mathematik und ihrer Grenzgebiete (3), 10. Springer-Verlag, Berlin, 1987. MR867684 (88f:53087)

2. Buckland, John A. Short-time existence of solutions to the cross curvature flow on 3manifolds. Proc. Amer. Math. Soc. 134, no. 6, 1803-1807, 2006. MR2207496 (2007a:35061)

3. Chow, Bennett and Hamilton, Richard S. The cross curvature flow of 3-manifolds with negative sectional curvature. Turk. J. Math. 28, 1-10, 2004. MR2055396 (2005a:53107)

4. Cao, Xiaodong; Ni, Yilong; Saloff-Coste, Laurent. Cross curvature flow on locally homogeneous three-manifolds. arXiV:0708.1922.

5. Farrell, F. Thomas and Ontaneda, Pedro. A caveat on the convergence of the Ricci flow for pinched negatively curved manifolds. Asian J. Math. 9, no. 3, 401-406, 2005. MR 2214959 (2006j:53097)

6. Farrell, F. Thomas and Ontaneda, Pedro. On the topology of the space of negatively curved metrics. arXiV:math.DG/0607367.

7. Glickenstein, David. Riemannian groupoids and solitons for three-dimensional homogeneous Ricci and cross curvature flows. arXiv:0710.1276.

8. Gromov, Mikhail and Thurston, William. Pinching constants for hyperbolic manifolds. Invent. Math. 89, no. 1, 1-12, 1987. MR892185 (88e:53058)

9. Guenther, Christine; Isenberg, James; Knopf, Dan. Stability of the Ricci flow at Ricci-flat metrics. Comm. Anal. Geom. 10, no. 4, 741-777, 2002. MR.1925501(2003g:53118)

10. Koiso, Norihito. On the second derivative of the total scalar curvature. Osaka J. Math. 16, no. 2, 413-421, 1979. MR.539596 (80j:53047)

11. Lunardi, Alessandra. Analytic semigroups and optimal regularity in parabolic problems. Progress in Nonlinear Differential Equations and Their Applications, 16. Birkhäuser Verlag, Basel, 1995. MR 1329547(96e:47039) 
12. Ma, Li and Chen, Dezhong. Examples for cross curvature flow on 3-manifolds. Calc. Var. Partial Differential Equations 26, no. 2, 227-243, 2006. MR2222245(2006m:53100)

13. Ye, Rugang. Ricci flow, Einstein metrics and space forms. Trans. Amer. Math. Soc. 338, no. 2, 871-896, 1993. MR1108615 (93j:58029)

Department of Mathematics, University of Texas at Austin, Austin, Texas 78713

E-mail address: danknopf@math.utexas.edu

Department of Mathematics, University of Texas at Austin, Austin, Texas 78713

Current address: Department of Mathematics, The University of Arizona, Tucson, Arizona 85721

E-mail address: ayoung@math.arizona.edu 